\title{
El desarrollo de valores de referencia para el perímetro braquial \\ según la estatura y su comparación \\ con otros indicadores utilizados para el tamizaje del estado nutricional ${ }^{1}$
}

\author{
Z. Mei, ${ }^{2}$ L. M. Grummer-Strawn, ${ }^{2}$ M. de Onís ${ }^{3}$ y R. Yip ${ }^{4}$
}

RESUMEN Durante muchos años se han usado los valores de perimetro braquial inferiores a cierto límite como indice alternativo del estado nutricional de los menores de 5 años de edad en épocas de hambruna o crisis de refugiados y también como método adicional de tamizaje en situaciones normales. Sin embargo, recientemente se ha puesto en duda la independencia del perímetro braquial respecto de la edad y el sexo. Tras revisar las pruebas científicas en las que se basan el uso y la interpretación del perímetro braquial, un Comité de Expertos de la OMS recomendó nuevos valores de referencia de perímetro braquial según la edad en menores de 5 años. Sin embargo, en algunas situaciones es difícil evaluar la edad de un niño y en tales circunstancias el perímetro braquial según la altura puede ser una buena alternativa. La regla QUAC (del inglés Quaker arm circumference) para medir la altura es un medio sencillo para determinar el punto de corte del perímetro braquial correspondiente a una altura dada. Este artículo describe los valores de referencia del perímetro braquial y la construcción y uso del medidor QUAC, así como la utilización del método de curvas de características funcionales (receiver operating characteristic curve) para evaluar el rendimiento del perímetro braquial, el perímetro braquial según la edad y el perímetro braquial según la altura en la detección de niños malnutridos.

1 Se publicó en inglés en el Bulletin of the World Health Organization, Vol. 75, No. 4, 1997, con el título "The development of a MUAC-for-height reference, including a comparison to other nutritional status screening indicators". () Organización Mundial de la Salud, 1997.

2 Centros para el Control y la Prevención de Enfermedades (CDC), División de Nutrición y Actividad Física, Atlanta, Georgia, EUA. Pueden pedirse separatas de la versión original en inglés al autor a la siguiente dirección postal: Division of Nutrition and Physical Activity, CDC, Mailstop K-25, 4770 Buford Highway, Atlanta, GA, EUA.

3 Organización Mundial de la Salud, Unidad de Nutrición, 1211 Ginebra, Suiza.

4 Fondo de las Naciones Unidas para la Infancia, Java, Indonesia.
El perímetro braquial, medido en el punto medio de la parte proximal del brazo, se ha usado durante muchos años como índice del estado nutricional en situaciones como hambrunas o crisis de refugiados en las que es difícil determinar la altura y el peso (1-4). El perímetro braquial también se ha usado en situaciones normales como instrumento adicional de tamizaje, por su poder para predecir la mortalidad infantil (5-7).
Durante los últimos 30 años se utilizó para detectar malnutridos entre los menores de 5 años un único punto de corte, generalmente 12,5 o $13,0 \mathrm{~cm}$ de perímetro braquial. Este límite se obtuvo en los años sesenta a partir de observaciones de niños polacos normales, bien nutridos $(1,8,9)$. Sin embargo, recientemente se ha puesto en tela de juicio la idea de que el perímetro braquial es independiente de la edad y el sexo y se ha sugerido utilizar 
valores $z$ de perímetro braquial, ajustados respecto a edad y sexo, como mejor indicador del estado nutricional $(10,11)$. Un Comité de Expertos de la OMS ha recomendado usar en niños de 6 a 60 meses nuevos valores de referencia de perímetro braquial según la edad $(12,13)$.

Para facilitar el trabajo de campo de evaluación rápida del estado nutricional, durante los años sesenta se desarrolló un método para relacionar el perímetro braquial con la altura, mediante el uso de una regla métrica denominada medidor QUAC (del inglés Quaker Arm Circumference measuring stick, regla métrica cuáquera para el perímetro braquial) $(14,15)$. El método de medición con la regla QUAC, en el que se mide el perímetro braquial a nivel medio de la parte proximal del brazo y luego se compara este valor con la estatura del niño, es sencillo, barato, fácil de usar, independiente de que la edad sea o no correcta, y bastante fiable (16-21). Sin embargo, los valores de referencia del método QUAC que se usan actualmente se obtuvieron a partir de varias series de datos de perímetro braquial de niños polacos bien nutridos (9) y de datos estaturales de niños sanos de aldeas de Nigeria occidental $(14,18)$ o de datos específicos de país (19). Este artículo proporciona datos de referencia de perímetro braquial según la altura para uso internacional, basados en los mismos datos recomendados por un Comité de Expertos de la OMS como datos de referencia de perímetro braquial según la edad $(12,13)$. También se propone un medidor QUAC "estándar" que puede facilitar la comparación de datos entre países.

\section{MATERIALES Y MÉTODOS}

Para derivar los valores de referencia de perímetro braquial según la altura utilizamos los datos de crecimiento de niños de 6 meses a 9 años de edad correspondientes a la muestra con la que se realizaron la primera y segunda Encuesta Nacional de Salud y de Estudio de la Nutrición (NHANES I y NHANES II, según sus siglas en inglés)
$(22,23)$. Estas dos encuestas fueron llevadas a cabo por el Centro Nacional de Estadísticas Sanitarias (NCHS) estadounidense, para obtener datos de muestras representativas de la población civil no recluida de todo el país. En la encuesta NHANES I, realizada durante el período 1971-1974, se incluyeron personas de todas las edades entre 1 y 74 años; en la NHANES II, realizada durante 1976-1980, las edades incluidas abarcaron desde los 6 meses hasta los 74 años. Se ha publicado una descripción detallada de las muestras seleccionadas $(22,23)$. En total se realizaron 23808 determinaciones de perímetro braquial en una muestra combinada NHANES I y NHANES II de niños de 6 meses a 9 años.

Para estimar la mediana de los valores de perímetro braquial en un pequeño intervalo de estatura usamos el método de ajuste de curvas del análisis exploratorio de datos (la "línea resistente trigrupal") (24). Los datos se dividieron en tres subgrupos según la estatura, se calculó la mediana de perímetro braquial de cada subgrupo y se ajustó una recta de regresión a estas tres medianas. Utilizamos "ventanas" de estatura de $6 \mathrm{~cm}$, subdivididas en subgrupos de $2 \mathrm{~cm}$ de ventana. Por ejemplo, los perímetros braquiales de entre 77,0 y $82,9 \mathrm{~cm}$ se subdividieron en tres subgrupos de 77,0 a $78,9 \mathrm{~cm}, 79,0$ a $80,9 \mathrm{~cm}$ y 81,0 a $82,9 \mathrm{~cm}$, y se calculó la mediana de cada subgrupo. Luego se estimó la recta de regresión correspondiente a esas medianas y el valor de perímetro braquial que el modelo predice para una altura de $80,0 \mathrm{~cm}$ (el punto medio de la ventana de estaturas) (figura 1). Este procedimiento se repitió para cada ventana de estaturas de $6 \mathrm{~cm}$, con puntos medios a intervalos de 1,0 cm desde $65,0 \mathrm{~cm}$ hasta 145,0 $\mathrm{cm}$, es decir, un total de 81 veces. Con un polinomio de quinto grado se estimó una curva mediana alisada ajustada a las 81 medianas estimadas.

Mediante la curva mediana alisada calculamos el residuo correspondiente a cada dato y la desviación estándar para cada ventana de $6 \mathrm{~cm}$ con relación a la curva mediana alisada. La desviación estándar se calculó como raíz cuadrada del error cuadrático medio. Como la distribución de perímetros braquiales es sesgada, calculamos una desviación estándar superior solo con los residuos positivos y una desviación estándar inferior solo con los negativos. Luego se estimó una curva ajustada a las 81 desviaciones estándar

\begin{abstract}
FIGURA 1. Ilustración del procedimiento de análisis exploratorio de los datos. Cada + representa un dato, las medianas son los puntos - y se presenta también la recta de regresión para la ventana de $6 \mathrm{~cm}$ utilizada para estimar el perímetro braquial correspondiente al punto medio de la ventana de estatura
\end{abstract}

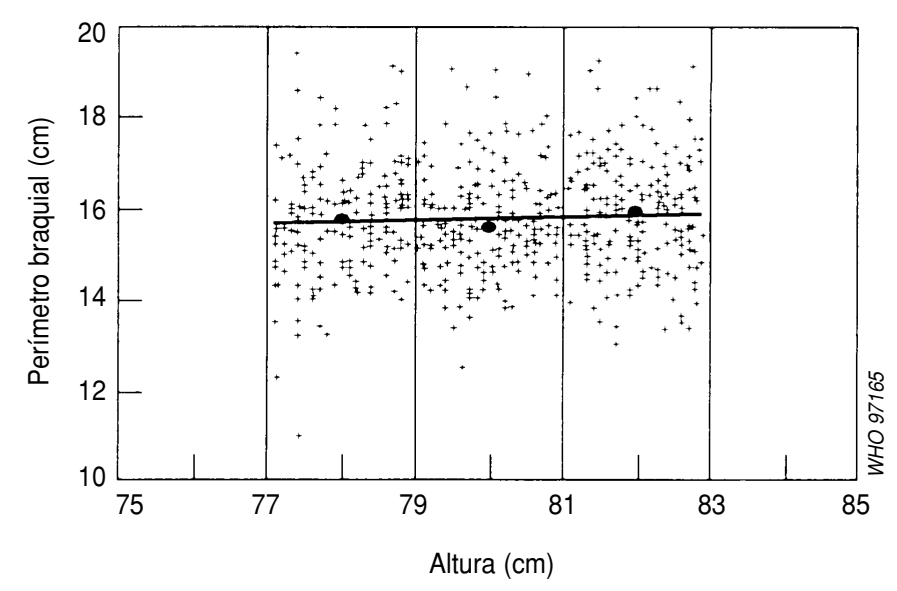


superiores e inferiores usando un polinomio de tercer grado.

Se calcularon así tres series de curvas de perímetro braquial según la altura: una para niños, otra para niñas y otra para los dos sexos combinados. Las curvas de desviación estándar en niños y en niñas resultaron similares y para mejorar la precisión se utilizaron para las tres series de curvas de referencia las curvas de desviación estándar para ambos sexos combinados.

Para eliminar cualquier discrepancia causada por la diferencia entre la determinación estatural en decúbito y en pie a lo largo del recorrido de alturas, todas las estaturas en decúbito de menos de $85 \mathrm{~cm}$ fueron ajustadas a estaturas en bipedestación mediante una substracción de 0,4 cm. Este ajuste de $4 \mathrm{~mm}$ se basó en la diferencia entre la medición de longitud y la medición de estatura en los datos de los niños de 24 a 35 meses de la encuesta NHANES II en los que se obtuvieron ambas medidas. Una vez efectuado el proceso de alisamiento, para los datos finales de referencia las alturas de menos de $85 \mathrm{~cm}$ fueron reconvertidas a longitudes mediante la adición de 0,4 cm. El resultado es que el protocolo de medida utilizado para obtener los valores de referencia es equivalente a los procedimientos estándar diseñados para tallar a los niños en el medio clínico o en trabajos de campo.

Se utilizaron datos procedentes de Sri Lanka (25), Nepal (26), Togo (27) y Malawi (28) para evaluar el rendimiento de los indicadores de perímetro braquial, perímetro braquial según la edad y perímetro braquial según la estatura, usando un valor $z<-2$ como estándar de referencia para considerar desnutrido a un niño. Las series de datos de Sri Lanka (1975-1976), Nepal (1975) y Togo (1976-1977) fueron obtenidas a partir de encuestas transversales nacionales realizadas con colaboración de los CDC y la Agencia de los Estados Unidos para el Desarrollo Internacional (USAID). Los tamaños de las muestras de niños de 6 a 59 meses de edad en esas tres encuestas fueron respectivamente 13469,6579 y 6120 . El Estudio de Nutrición Maternoinfan- til de Malawi es una encuesta longitudinal trienal (1986-1969) de 3009 registros de niños de 6 a 59 meses (28). Para evaluar el rendimiento de los nuevos indicadores utilizamos curvas de características funcionales (curvas ROC - de receiver operating characteristic curve, según su denominacion original en inglés-) (29) en las que se representa en abscisas 1 - especificidad y en ordenadas, la sensibilidad. En general, cuanto mayor es la desviación de la curva ROC de características funcionales de la línea de azar (que conecta las dos esquinas del gráfico), mejor es el rendimiento del indicador.

\section{RESULTADOS}

El cuadro 1 presenta los valores de referencia para niños, niñas y ambos sexos combinados, para el grupo de 6 a 119 meses de edad con alturas entre 65 y $145 \mathrm{~cm}$, según los datos de NHANES I y NHANES II. Por limitaciones de tamaño muestral los datos de referencia no incluyen niños de menos de $65 \mathrm{~cm}$ ni de más de $145 \mathrm{~cm}$ de altura. Las figuras 2, 3 y 4 son las gráficas de crecimiento del perímetro braquial basadas en los datos del cuadro 1. Las curvas corresponden a la mediana y a 1,0, 2,0 y 3,0 desviaciones estándar por encima y por debajo de la mediana. En las gráficas hay una pequeñísima ruptura correspondiente a la discontinuidad entre la longitud en decúbito y la estatura en bipedestación (unos 0,01 cm de perímetro braquial) a los $85 \mathrm{~cm}$. Las curvas específicas de edad recogen la mayor parte de la variación relacionada con el punto de corte fijo, pero hay también diferencias significativas entre niños y niñas en los intervalos de alturas de 65,0 a 80,0 cm y de 120,0 a $145,0 \mathrm{~cm}$.

El anexo 1 muestra el programa SAS (Statistical Analysis System) que se usó para calcular la curva mediana y las curvas de desviación estándar superior e inferior. Utilizando este programa con sus propios datos, los lectores podrán asignar fácilmente valores $z$ a cada niño.
La figura 5 presenta las curvas de características funcionales (curvas ROC) correspondientes a perímetro braquial, perímetro braquial según la edad y perímetro braquial según la altura, para detectar niños malnutridos de 6 a 59 meses de edad en Sri Lanka. En la detección de niños malnutridos el perímetro según la altura y el perímetro según la edad tuvieron mayor sensibilidad y especificidad que un valor fijo de perímetro braquial. Con los datos de estudios nutricionales de Nepal y Togo y con los del estudio longitudinal de Malawi se obtuvieron resultados similares en cuanto a curvas de características funcionales de los tres indicadores (pueden solicitarse los resultados a los autores).

\section{DISCUSIÓN}

Este trabajo presenta una serie de curvas de referencia para el perímetro braquial según la estatura y muestra que los valores $z$ de perímetro braquial según la altura y de perímetro braquial según la edad son mejores predictores del peso según la altura que un valor fijo de cualquiera de los perímetros braquiales. El perímetro según la altura tiene algunas ventajas respecto del peso para la talla en situaciones de crisis de emergencias o refugiados: no requiere el uso del escalas complicadas y sufre menos el efecto de errores en la medición de la estatura. Por ejemplo, considérese un niño cuya altura, peso y perímetro braquial verdaderos son 100,0 cm, 12,6 kg y 14,1 $\mathrm{cm}$, respectivamente. Su peso normalizado según la altura es $z=-2,29$ de acuerdo con los valores de referencia internacionales NCHS/OMS de crecimiento (30); su perímetro braquial normalizado correspondiente a su altura es $z=-2,29$ según los datos aquí expuestos. En condiciones de crisis puede ser difícil disponer de una plataforma apropiada de medición, completamente plana y con una pared vertical adyacente. En tales circunstancias, o si la estatura se mide con poco cuidado y se registra, por ejem- 
CUADRO 1. Valores de referencia de perímetro braquial según la longitud o la estatura. Valores correspondientes a la mediana de la distribución y a 2 y 3 desviaciones estándar (DE) por debajo de la mediana

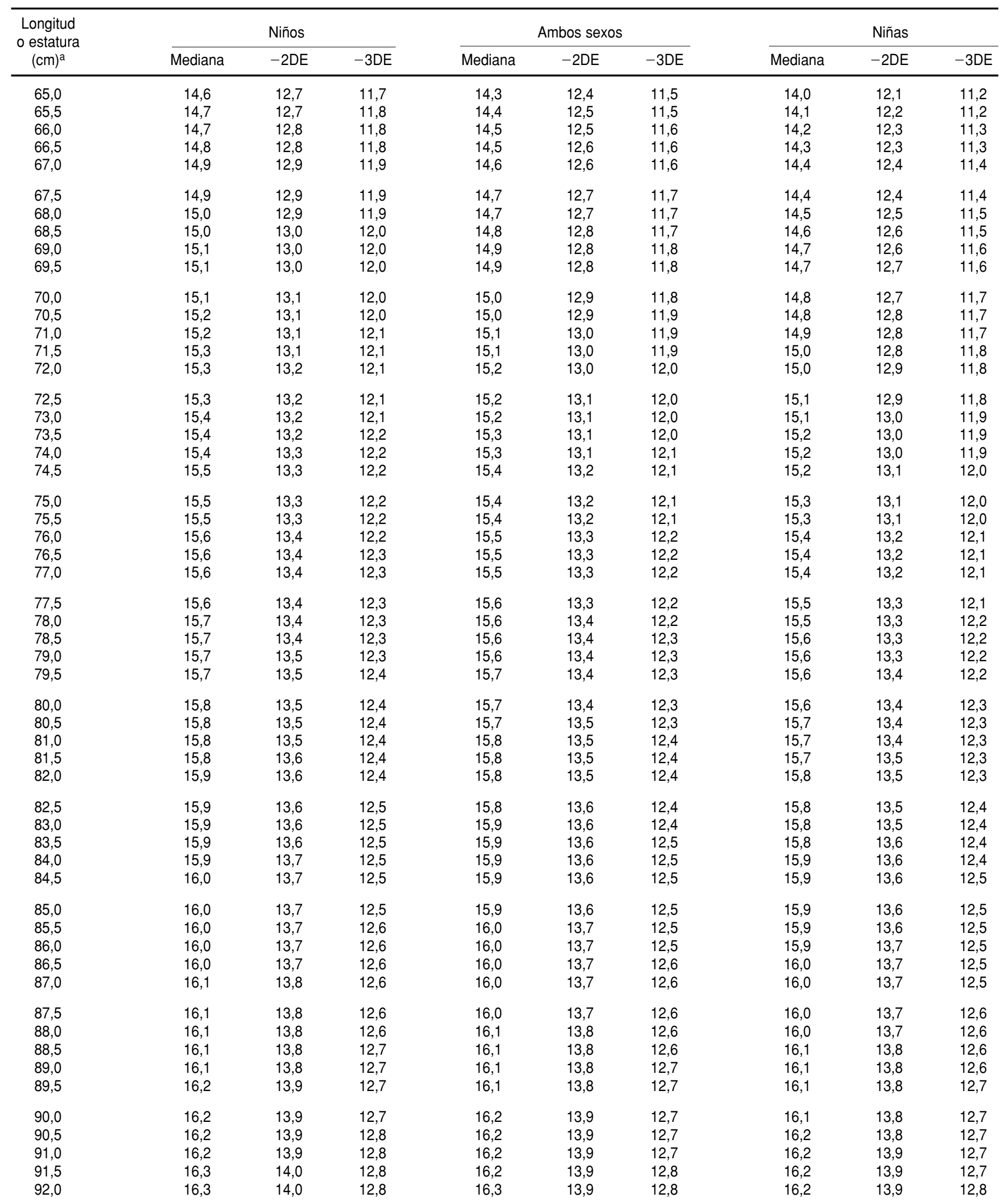


CUADRO 1. (Continuación)

\begin{tabular}{|c|c|c|c|c|c|c|c|c|c|}
\hline $\begin{array}{l}\text { Longitud } \\
\text { o estatura } \\
(\mathrm{cm})^{\mathrm{a}}\end{array}$ & \multicolumn{3}{|c|}{ Niños } & \multicolumn{3}{|c|}{ Ambos sexos } & \multicolumn{3}{|c|}{ Niñas } \\
\hline 92,5 & 16,3 & 14,0 & 12,9 & 16,3 & 14,0 & 12,8 & 16,2 & 13,9 & 12,8 \\
\hline 93,5 & 16,4 & 14,1 & 12,9 & 16,3 & 14,0 & 12,9 & 16,3 & 14,0 & 12,8 \\
\hline 94,0 & 16,4 & 14,1 & 12,9 & 16,4 & 14,0 & 12,9 & 16,3 & 14,0 & 12,8 \\
\hline 94,5 & 16,4 & 14,1 & 13,0 & 16,4 & 14,1 & 12,9 & 16,3 & 14,0 & 12,9 \\
\hline 95,5 & 16,5 & 14,2 & 13,0 & 16,4 & 14,1 & 13,0 & 16,4 & 14,1 & 12,9 \\
\hline 96,0 & 16,5 & 14,2 & 13,0 & 16,5 & 14,1 & 13,0 & 16,4 & 14,1 & 12,9 \\
\hline 96,5 & 16,5 & 14,2 & 13,1 & 16,5 & 14,2 & 13,0 & 16,4 & 14,1 & 13,0 \\
\hline 97,0 & 16,6 & 14,2 & 13,1 & 16,5 & 14,2 & 13,0 & 16,5 & 14,1 & 13,0 \\
\hline 97,5 & 16,6 & 14,3 & 13,1 & 16,5 & 14,2 & 13,1 & 16,5 & 14,2 & 13,0 \\
\hline 98,0 & 16,6 & 14,3 & 13,1 & 16,6 & 14,2 & 13,1 & 16,5 & 14,2 & 13,0 \\
\hline 100,5 & 16,8 & 14,4 & 13,3 & 16,7 & 14,4 & 13,2 & 16,7 & 14,3 & 13,2 \\
\hline 101,0 & 16,8 & 14,5 & 13,3 & 16,7 & 14,4 & 13,2 & 16,7 & 14,4 & 13,2 \\
\hline 101,5 & 16,8 & 14,5 & 13,3 & 16,8 & 14,4 & 13,3 & 16,7 & 14,4 & 13,2 \\
\hline 102,0 & 16,9 & 14,5 & 13,4 & 16,8 & 14,5 & 13,3 & 16,7 & 14,4 & 13,2 \\
\hline 102,5 & 16,9 & 14,6 & 13,4 & 16,8 & 14,5 & 13,3 & 16,8 & 14,4 & 13,3 \\
\hline 103,0 & 16,9 & 14,6 & 13,4 & 16,9 & 14,5 & 13,4 & 16,8 & 14,5 & 13,3 \\
\hline 103,5 & 16,9 & 14,6 & 13,4 & 16,9 & 14,6 & 13,4 & 16,8 & 14,5 & 13,3 \\
\hline 104,0 & 17,0 & 14,6 & 13,5 & 16,9 & 14,6 & 13,4 & 16,9 & 14,5 & 13,4 \\
\hline 104,5 & 17,0 & 14,7 & 13,5 & 17,0 & 14,6 & 13,4 & 16,9 & 14,6 & 13,4 \\
\hline 105,0 & 17,0 & 14,7 & 13,5 & 17,0 & 14,6 & 13,5 & 16,9 & 14,6 & 13,4 \\
\hline 105,5 & 17,1 & 14,7 & 13,6 & 17,0 & 14,7 & 13,5 & 17,0 & 14,6 & 13,4 \\
\hline 110,0 & 17,4 & 15,0 & 13,8 & 17,4 & 15,0 & 13,8 & 17,3 & 14,9 & 13,7 \\
\hline 110,5 & 17,4 & 15,0 & 13,8 & 17,4 & 15,0 & 13,8 & 17,3 & 14,9 & 13,7 \\
\hline 111,0 & 17,5 & 15,1 & 13,9 & 17,4 & 15,0 & 13,8 & 17,4 & 15,0 & 13,8 \\
\hline 111,5 & 17,5 & 15,1 & 13,9 & 17,5 & 15,0 & 13,8 & 17,4 & 14,0 & 13,8 \\
\hline 112,0 & 17,5 & 15,1 & 13,9 & 17,5 & 15,1 & 13,9 & 17,5 & 15,0 & 13,8 \\
\hline 112,5 & 17,6 & 15,1 & 13,9 & 17,6 & 15,1 & 13,9 & 17,5 & 15,1 & 13,9 \\
\hline 113,0 & 17,6 & 15,2 & 14,0 & 17,6 & 15,1 & 13,9 & 17,6 & 15,1 & 13,9 \\
\hline 113,5 & 17,7 & 15,2 & 14,0 & 17,6 & 15,2 & 14,0 & 17,6 & 15,2 & 13,9 \\
\hline 114,0 & 17,7 & 15,2 & 14,0 & 17,7 & 15,2 & 14,0 & 17,7 & 15,2 & 14,0 \\
\hline 114,5 & 17,7 & 15,3 & 14,0 & 17,7 & 15,2 & 14,0 & 17,7 & 15,2 & 14,0 \\
\hline 115,0 & 17,8 & 15,3 & 14,0 & 17,8 & 15,3 & 14,0 & 17,8 & 15,3 & 14,0 \\
\hline 115,5 & 17,8 & 15,3 & 14,1 & 17,8 & 15,3 & 14,1 & 17,8 & 15,3 & 14,1 \\
\hline 116,0 & 17,9 & 15,4 & 14,1 & 17,9 & 15,3 & 14,1 & 17,9 & 15,3 & 14,1 \\
\hline 116,5 & 17,9 & 15,4 & 14,1 & 17,9 & 15,4 & 14,1 & 17,9 & 15,4 & 14,1 \\
\hline 117,0 & 18,0 & 15,4 & 14,1 & 18,0 & 15,4 & 14,1 & 18,0 & 15,4 & 14,1 \\
\hline 117,5 & 18,0 & 15,4 & 14,2 & 18,0 & 15,4 & 14,2 & 18,0 & 15,5 & 14,2 \\
\hline 118,0 & 18,0 & 15,5 & 14,2 & 18,1 & 15,5 & 14,2 & 18,1 & 15,5 & 14,2 \\
\hline 118,5 & 18,1 & 15,5 & 14,2 & 18,1 & 15,5 & 14,2 & 18,1 & 15,5 & 14,2 \\
\hline 119,0 & 18,1 & 15,5 & 14,2 & 18,2 & 15,6 & 14,3 & 18,2 & 15,6 & 14,3 \\
\hline 119,5 & 18,2 & 15,6 & 14,2 & 18,2 & 15,6 & 14,3 & 18,2 & 15,6 & 14,3 \\
\hline
\end{tabular}


CUADRO 1. (Continuación)

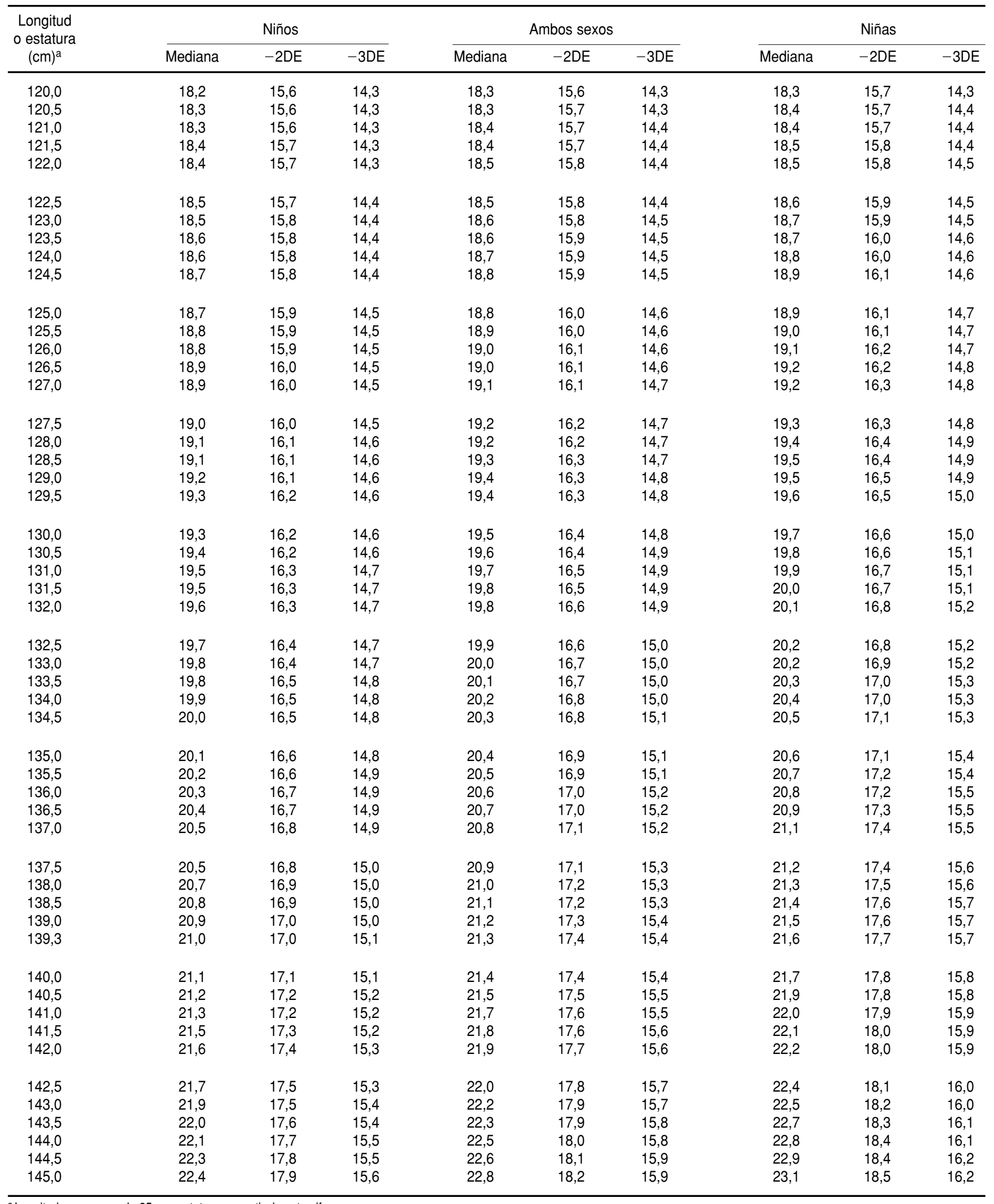

a Longitudes menores de $85 \mathrm{~cm}$; estaturas a partir de esta cifra. 
FIGURA 2. Curvas de referencia de perímetro braquial según altura para niños de alturas entre 65 y $145 \mathrm{~cm}$. Valores correspondientes a la mediana de la distribución y a una 0 varias desviaciones estándar por encima o debajo de la mediana

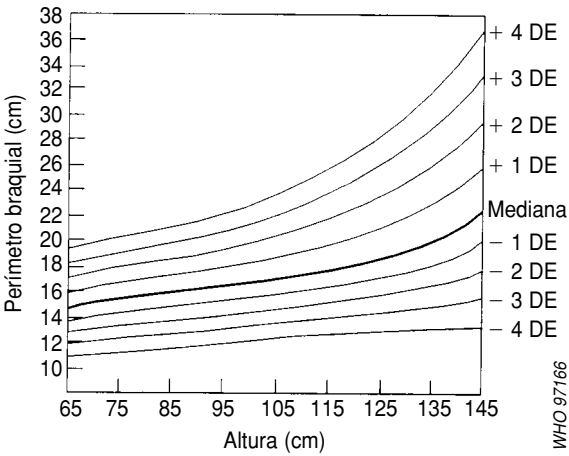

plo, un valor $3 \mathrm{~cm}$ menor que el verdadero, el valor $z$ de peso para edad aumenta a $-1,81$, mientras que el valor $z$ de perímetro braquial $(-2,15)$ apenas cambia. En realidad es más probable que la propia medición del perímetro braquial sea imprecisa por no tomarse exactamente la circunferencia del punto medio de la parte proximal del brazo, porque el brazo esté flácido o porque la cinta se apriete demasiado o no se ajuste bien.

Para estimar los valores de referencia de perímetro braquial según la altura se ajustó la longitud en decúbito a la estatura en pie. Sin embargo, las diferencias entre los perímetros correspondientes a la longitud en decúbito y a la estatura en pie son muy pequeñas (alrededor de 0,01 cm) y no tienen significación clínica. En términos prácticos, esto significa que da igual utilizar
FIGURA 3. Curvas de referencia de perímetro braquial según la altura para niñas de alturas entre 65 y $145 \mathrm{~cm}$. Valores correspondientes a la mediana de la distribución y a una 0 varias desviaciones estándar por encima o debajo de la mediana

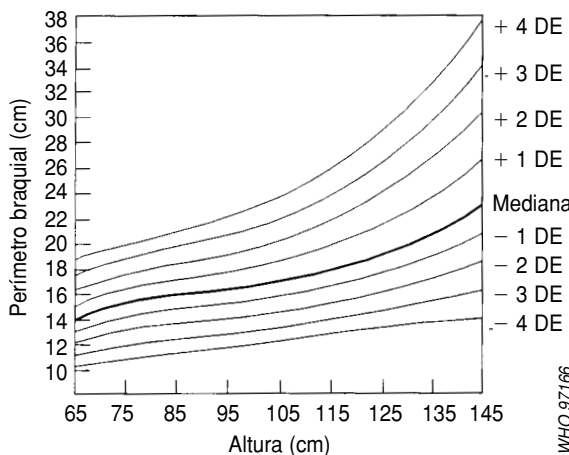

la longitud en decúbito o la estatura en bipedestación. Sin embargo, recomendamos que los niños que miden hasta $85 \mathrm{~cm}$ sean tallados en decúbito y que los de mayor estatura sean tallados en pie, para seguir las normas internacionales (12) y los protocolos actuales de talla para la edad o altura para la talla.

El medidor QUAC puede usarse como método rápido para determinar niveles de nutrición en grandes poblaciones y como instrumento de detección selectiva de la malnutrición en niños $(14,15)$. Tiene muchas ventajas prácticas, especialmente cuando las condiciones de campo son difíciles. El anexo 2 describe detalladamente la construcción y el uso de un medidor QUAC modificado. Los datos de referencia descritos en este artículo han de utilizarse con dicho medidor QUAC modificado.

\section{REFERENCIAS}

1. Jellife DB, Jellife EFP. The arm circumference as a public health index of protein-calorie malnutrition in early childhood. J Trop Pediatr 1969;15:177-260.

2. Velzeboer MI, et al. Evaluation of arm circumference as a public health index of proteincalorie malnutrition in early childhood. J Trop Pediatr 1983;29:135-144.

3. Shakir A. Arm circumference in the surveillance of protein-energy malnutrition in Baghdad. Am J Clin Nutr 1975;26:661-665.

4. McDowell I, King FS. Interpretation of arm circumference as an indicator of nutritional status. Arch Dis Child 1982;57:229-296.

5. Chen LC, Chowdhury A, Huffman SL. Anthropometric assessment of energy protein malnutrition and subsequent risk of mortality among preschool-aged children. Am J Clin Nutr 1980;33:1836-1845.

6. Trowbridge FL, Sommer A. Nutritional anthropometry and mortality risk. Am J Clin Nutr 1981;34:2591-2592.

7. Briend A, Wojtyniak B, Rowlands MG. Arm circumference and other factors in children at high risk of death in rural Bangladesh. Lancet 1987:2:725-728.

8. Burgess HJL, Burgess AP. A modified standard for mid-upper arm circumference in
FIGURA 4. Curvas de referencia de perímetro braquial según altura para niños de ambos sexos y altura entre 65 y $145 \mathrm{~cm}$. Valores correspondientes a la mediana de la distribución y a una 0 varias desviaciones estándar por encima o debajo de la mediana

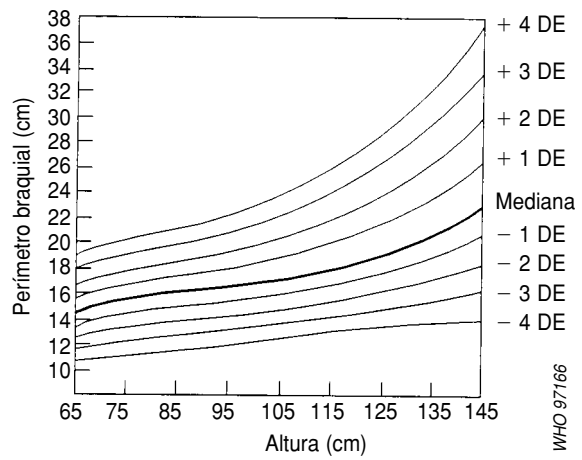

FIGURA 5. Comparación de la utilidad de las curvas de características funcionales (curvas ROC) del perímetro braquial según la edad y del perímetro braquial según la altura para detectar malnutrición (definida por un peso según la estatura < $-2 \mathrm{DE}$ ) en niños de Sri Lanka de 6 a 59 meses de edad, 1975-1976. La diagonal representa la línea de azar para la curva de características funcionales

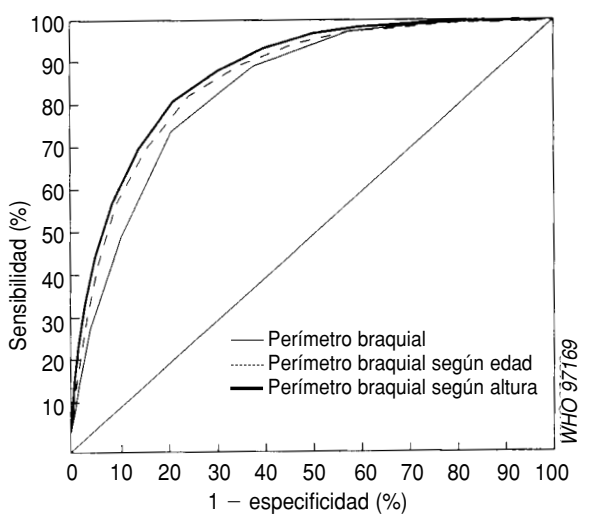

young children-Monograph 8. J Trop Pediatr 1969:15:189-192

9. Jellife DB. The assesment of the nutritional status of the community. Geneva: World Health Organization; 1966. (WHO Monograph Series 53).

10. Hall G, Chowdhury S, Bloem M. Use of midupper-arm circumference $z$-scores in nutritional assesment. Lancet 1993;341:1481.

11. Van den Broeck J, Eeckels R, Vuylsteke J. Influence of nutritional status on child mortality in rural Zaire. Lancet 1993;341:1491-1495.

12. World Health Organization. Physical status: The use and interpretation of anthropometry. Report of 
a WHO Expert Committee. Geneva: WHO; 1995. (WHO Technical Report Series 854).

13. de Onis M, Yip R, Mei Z. The development of MUAC-for-age reference data recommended by a WHO Expert Committte. Bull World Health Organ 1997;75:11-18.

14. Arnold R. The arm circumference as a public health index of protein-calorie malnutrition of early childhood: XVIII-The QUAC stick: A field measure used by the Quaker Service Team in Nigeria. J Trop Pediatr 1969;15:243-247.

15. Davis LE. Epidemiology of famine in the Nigerian crisis: Rapid evaluation of malnutrition by height and arm circumference in large populations. Am J Clin Nutr 1971;24: 358-364.

16. Amdekar YK, et al. "QUAC-stick"-field measure for quick assesment of nutritional status of preschool children. Indian Pediatr 1972;9: 397-402.

17. Jellife DB, Jellife EFP. Age-independent anthropometry. Am J Clin Nutr 1971;24: 1377-1379.

18. Shakir A. QUAC stick in the assesment of protein-calorie malnutrition in Baghdad. Lancet 1973;1:762-764.
19. Anderson MA. Use of height-arm circumference measurement for nutritional selectivity in Sri Lanka school feeding. Am J Clin Nutr 1975;28:775-781.

20. Sommer A, Loewenstein MS. Nutritional status and mortality: A prospective validation of the QUAC stick. Am J Clin Nutr 1975;28: 287-292.

21. Brown RC. Nutrition surveillance by QUAC stick. Trans Roy Soc Trop Med Hyg 1987;81: 1038-1039.

22. National Center for Health Statistics. Plan and operation of the Health and Nutrition Examination Survey, United States, 1971-1973. Rockville, MD: NCHS; 1973. (Vital and Health Statistics, Series 1, No. 10).

23. McDowell A, et al. Plan and operation of the second National Health and Nutrition Examination Survey, United States, 1976-1980. Rockville, MD: National Center for Health Statistics; 1981. (Vital and Health Statistics, Series 1, No. 15).

24. Velleman PK, Hoaglin DC. Applications, basics, and computing of exploratory data analysis. Boston: Duxbury; 1981.
25. Office of Nutrition, United States Agency for International Development. Sri Lanka Nutrition Status Survey 1976. Washington, DC: USAID; 1978.

26. Office of Nutrition, United States Agency for International Development. Nepal nutrition status survey 1975. Washington, DC: USAID; 1978.

27. Office of Nutrition, United States Agency for International Development. Togo Nutrition Status Survey 1977. Washington, DC: USAID; 1978.

28. Pelletier DL, et al. Child anthropometry and mortality in Malawi: Testing for effect modification by age and length of follow-up and confounding by socioeconomic factors. J. Nutr 1994; 124:2082S-2105S.

29. Metz CE. Basic principle of ROC analysis. Semin Nucl Med 1978;8:283-298.

30. World Health Organization. Measuring change in nutritional status. Geneva: WHO; 1983.

\section{ANEXO 1. Programa SAS para obtener valores de referencia de perímetro braquial según la estatura (longitud)}

ALT es la altura o la longitud del niño (en $\mathrm{cm}$ ). PB es la longitud del perímetro braquial medido en el punto medio del brazo y PBALT es el valor $z$ del perímetro según la altura. ${ }^{5}$

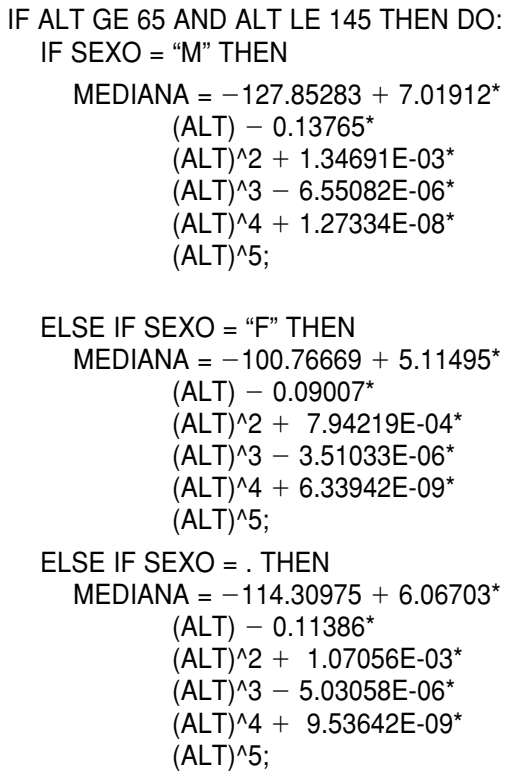

5 Téngase en cuenta que este programa para SAS contiene palabras en inglés como IF o THEN que son a la vez funciones lógicas del SAS y que por tanto no pueden traducirse. En cambio las variables definidas por el usuario pueden nombrarse arbitrariamente y aquí se han usado por ejemplo SEXO, PB y PBALT para lo que en inglés era SEX, MUAC (mid-upper-arm circumference) y MUACHTZ (MUAC-for-height), respectivamente. (N. del t.) 


\section{ANEXO 2. Construcción y uso del medidor QUAC modificado}

1. Constrúyase un medidor QUAC como el que aparece en la figura 1, con un listón grueso de madera de unos $150 \mathrm{~cm}$ de largo y $3 \times 3 \mathrm{~cm}$ de espesor. En una cara del listón háganse marcas cada $0,5 \mathrm{~cm}$, desde 0 en un extremo hasta 145 cerca del otro. Escríbanse las longitudes correspondientes a intervalos de $1 \mathrm{~cm}$, como muestra la figura. En una cara del listón adyacente a la de la escala de altura han de marcarse los valores de referencia correspondientes a la mediana menos dos desviaciones estándar de perímetro braquial según la altura (véase cuadro 1). En la otra cara del listón adyacente a la escala de medición han de marcarse los valores correspondientes a la mediana menos 3 desviaciones estándar.

Este listón es apropiado para medir la altura de niños de más de $85 \mathrm{~cm}$ (los de menor altura han de ser tallados sobre una mesa métrica). Es preferible preparar dos medidores, uno para niños y otro para niñas; o si no, puede prepararse un único listón en el que se usen los valores para ambos sexos combinados.

2. Colóquese el listón bien sujeto firme y verticalmente sobre una plataforma lisa contra una pared vertical. El niño ha de ponerse bien derecho en pie con su espalda apoyada contra el listón. La altura se mide usando una regla plana.

3. Mídase el perímetro braquial en $\mathrm{mm}$ y véase si el valor es menor que el que aparece en la cara correspondiente a la mediana menos $3 \mathrm{DE}$; si lo es, se trata de un caso de desnutrición grave. $\mathrm{Si}$ no lo es, mírese en la cara correspondiente a mediana menos $2 \mathrm{DE}$. Si el valor es menor que este, clasifíquese al niño como caso de desnutri- ción moderada; si es mayor, clasifíquese al niño como no desnutrido. De esta forma todos los niños son clasificados con un grado de desnutrición grave o moderada o sin desnutrición, de la misma manera que con el método de peso según la estatura.

FIGURA A1. Medidor QUAC modificado según la OMS

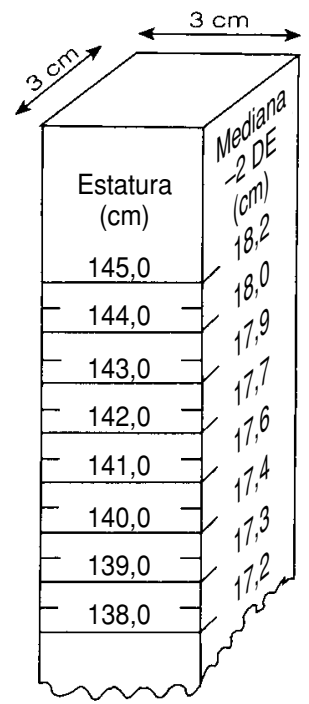

ABSTRACT Mid-upper-arm circumference (MUAC) based on a single cut-off value for all children under 5 years of age has been used for many years as an alternative nutritional status index for children during famines or refugee crises, and as an additional screening tool in nonemergencies. However, it has recently been questioned whether MUAC is age- and sex-independent. After reviewing the scientific evidence underlying the use and interpretation of MUAC, a WHO Expert Committee recommended a new MUAC-for-age reference for under-5-year-olds. In some settings, however, it is difficult to assess a child's age and in such circumstances MUAC-for height may be a good alternative. The height-based QUAC stick offers a simple means of adjusting MUAC cut-offs according to height, and the MUAC-for-height reference and construction and use of the QUAC stick are described in this article. Also described is the use of the receiver operating characteristic (ROC) curve method to evaluate the performance of MUAC, MUAC-for-age, and MUAC-for-height in screening malnourished children. 